\title{
Silicon-on-insulator technology for microelectromechanical applications
}

\author{
A. Y. Usenko, W. N. Carr \\ New Jersey Microsystems, Inc. \\ 240 King Blvd., Newark, NJ 07102,njm@technologist.com
}

\begin{abstract}
A purpose of the paper is to give a review of recent development (1998-1999) in microelectromechanical (MEMS) devices formed on silicon-on-insulator (SOI) substrates. Advantages of using SOI are summarised. Problems of CMOS-MEMS integration for smart sensors are listed. Examples of successful use of SOI to fabricate advanced MEMS are given and future prospects MEMS on SOI are evaluated.
\end{abstract}

Keywords: micromechanical systems, silicon-on-insulator, sensors, review.

Paper received 29.12.98; revised manuscript received 15.03.99; accepted for publication 22.03.99.

SOI is an extremely versatile starting material for fabricating micromechanical systems both with and without the inclusion of silicon integrated circuits on a shared substrate. SOI permits a means of structuring starting sandwich materials to provide a highly precise control of several material parameters in addition to permitting unique structures not easy to obtain without SOI technology. The SOI technology referred to in this paper extends beyond using silicon oxide as the dielectric to include customized dielectrics and substrates. We use this broad definition of SOI because that is precisely the direction that this technology will evolve as CMOS and bipolar integrated circuits are mated with MEMS in costeffective and technology-enhanced ways.

The utilization of SOI for MEMS applications is summarized in the Table with respect to SOI structure and resulting advantages of performance, parameter specification, and processing. This summary does not permit detailing each structure since there are a large number of detail structures that will evolve from the marriage of MEMS with SOI technology.

A fundamental advantage of the SOI structure is that a thin surface film is obtained in which the film can be tailored by preprocessing of the source wafer to provide a precise film thickness, modulus, and doping properties. The SOI technology permits tailoring mechanical properties in addition to electrical properties in a very uniform manner. This basic process point provides a fundamental advantage to the use of SOI starting material in MEMS applications. In MEMS, warping of released structures is often a major problem especially when the released structure is of large area and thin. If thermistors or heating elements are incorporated into the released structure the advantage of using a bulk-derived film provides an important increase of uniformity in resistivity and resulting production yield.

For bimorph and multimorph surface structures the single, uniform film underlying CVD, evaporated, epitaxial, etc. films contributes to the overall uniformity of mechanical and electrical characteristics of the completed sensor or actuator structure. The increased tensile breaking (and plastic deformation threshold) of single crystal films invariably exhibit increased mechanical strength in a finished structure. Many flexed MEMS structures are improved by using films of increased tensile breaking stress.

The mechanical and electrical isolation that can be obtained by etching from either the topside or the back- 


\section{A. Y. Usenko et. al.: Silicon-on-insulator technology for ...}

Table. SOI-MEMS technology utilization summary.

\begin{tabular}{|c|c|c|c|}
\hline SOI Structure & Performance & Parameter & Process \\
\hline $\begin{array}{l}\text { Released monomorph } \\
\text { micro platform, } \\
\text { cantilevers, beams as } \\
\text { structural members, } \\
\text { sensors, and actuators }\end{array}$ & $\begin{array}{l}\text { More precise fabrication } \\
\text { control }\end{array}$ & $\begin{array}{l}\text { Reduced warping, controlled } \\
\text { resistivity, uniform thickness, } \\
\text { minimal internal stress, } \\
\text { increased tensile breaking } \\
\text { stress }\end{array}$ & $\begin{array}{l}\text { Ease of film release } \\
\text { The bonding } \\
\text { dielectric becomes } \\
\text { the sacrificial layer. }\end{array}$ \\
\hline $\begin{array}{l}\text { Released bimorph } \\
\text { actuators and sensors }\end{array}$ & $\begin{array}{l}\text { More narrow windows for } \\
\text { sensitivities and actuation } \\
\text { efficiency }\end{array}$ & $\begin{array}{l}\text { Precision thickness, repeatable } \\
\text { internal stress, higher } \\
\text { deflection limits }\end{array}$ & $\begin{array}{l}\text { Reduced yield } \\
\text { spread } \\
\text { requirement for } \\
\text { deposited films }\end{array}$ \\
\hline $\begin{array}{l}\text { Internal dielectric etch } \\
\text { stop }\end{array}$ & $\begin{array}{l}\text { More narrow performance } \\
\text { window }\end{array}$ & $\begin{array}{l}\text { Precise control of } \\
\text { semiconductor film thickness } \\
\text { used with backside/frontside } \\
\text { etching }\end{array}$ & $\begin{array}{l}\text { Convenient etch } \\
\text { stop for TMAH, } \\
\text { EDP, KOH }\end{array}$ \\
\hline $\begin{array}{l}\text { Integrated circuits } \\
\text { with MEMS on the } \\
\text { same substrate }\end{array}$ & $\begin{array}{l}\text { Higher speed, reduced } \\
\text { spurious signal pickup, } \\
\text { higher sensitivity }\end{array}$ & $\begin{array}{l}\text { Reduced circuit capacitance, } \\
\text { reduced magnetic pickup area, } \\
\text { higher functional density }\end{array}$ & $\begin{array}{l}\text { SOI is basically } \\
\text { compatible with } \\
\text { MEMS }\end{array}$ \\
\hline $\begin{array}{l}\text { Surface films } \\
\text { customized using FZ, } \\
\text { compensated CZ, and } \\
\text { other preprocesses }\end{array}$ & $\begin{array}{l}\text { Unique performance } \\
\text { sensors }\end{array}$ & $\begin{array}{l}\text { Unique parameters tailored } \\
\text { for optical and IR elements, } \\
\text { magnetometers, PIN diodes, } \\
\text { bolometers }\end{array}$ & $\begin{array}{l}\text { Obtainable only } \\
\text { with SOI-type } \\
\text { processing in } \\
\text { many cases }\end{array}$ \\
\hline $\begin{array}{l}\text { Customized dielectrics } \\
\text { instead of } \mathrm{SiO}_{2}\end{array}$ & $\begin{array}{l}\text { Tailoring of the dielectric } \\
\text { constant and optical } \\
\text { bandpass, for example }\end{array}$ & $\begin{array}{l}\text { Control dielectric loss vector, } \\
\text { permittivity, optical and IR } \\
\text { index, thermal conductivity }\end{array}$ & $\begin{array}{l}\text { Utilizes developed } \\
\text { wafer bonding } \\
\text { equipment }\end{array}$ \\
\hline Customized substrates & $\begin{array}{l}\text { Mechanical strength, } \\
\text { optical transparency, } \\
\text { thickness and thermal } \\
\text { conductivity. Relates to } \\
\text { packaging importantly }\end{array}$ & $\begin{array}{l}\text { Control Young's modulus, } \\
\text { permittivity, tensile breaking } \\
\text { stress }\end{array}$ & $\begin{array}{l}\text { Generally does not } \\
\text { complicate } \\
\text { processing }\end{array}$ \\
\hline
\end{tabular}

side of SOI wafers permits a variety of structures in which the dimensions can be precisely controlled. These dimensions are precisely controlled vertically by using the dielectric film within the SOI sandwich as an etch stop. In these processing situations the silicon is removed using a timed RIE process step or any combination of variety of anisotropic etchants including $\mathrm{KOH}$, TMAH, EDP, and $\mathrm{XeF}_{6}$.

The integration of CMOS and bipolar circuits onto a shared substrate with MEMS is the subject of considerable efforts worldwide. Using SOI an added opportunity exists to process CMOS or MEMS prior to SOI wafer bonding to obtain certain performance advantages. Most methodologies have resulted in integrated circuits being fabricated first where MEMS processing is a final process sequence with the release of MEMS structures.

When surface films of SOI starting materials are used as sensors it is possible to obtain an order of magnitude improvement in uniformity over deposited films. The uniformity advantage becomes even more important for large arrays fabricated using SOI structures. A similar customization can be obtained by selecting dielectrics other than $\mathrm{SiO}_{2}$ as the dielectric sandwich materials. The dielectric can be tailored with a low dielectric constant to obtain improved performance of strip transmission lines in millimeter wave network applications, for example.

\section{Examples of successful MEMS with SOI}

Wafer bonding allows a new degree of freedom in design and fabrication of SOI material combinations $[1,2,3]$ that previously would have been excluded because these material combinations cannot be realized by the conventional approach of epitaxial growth.

In [4] an SOI-based microelectromechanical quartz rate sensor is presented. These techniques have been adapted to applications in the aerospace, military, industrial, medical, telecommunications and automotive industries to realize miniaturized highly integrated MEMS in a cost-effective manner.

Studies on $\mathrm{SiO}_{2}-\mathrm{SiO}_{2}$ bonding with a hydrofluoric acid 
A. Y. Usenko et. al.: Silicon-on-insulator technology for ...

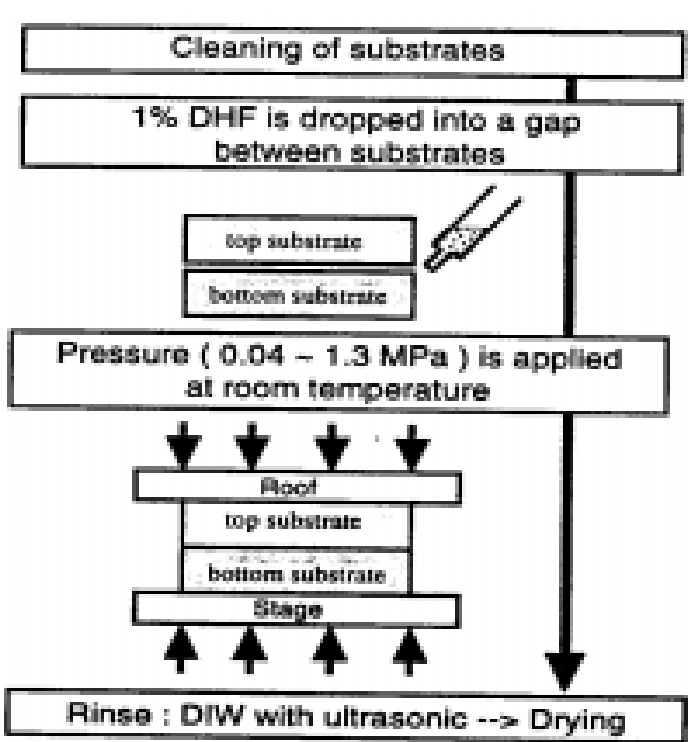

Fig. 1. Procedure of low temperature bonding for MEMS packaging [5].

(HF) treated surface in [5] show a remarkable feature that bonding can be obtained at room temperature. Advantages of this method are low thermal damage, low residual stress and simplicity of the bonding process, which are expected for the packaging and assembly of MEMS, Fig. 1.

MEMS can build unique 3-D structures for critical RF (radiofrequency) components, such as suspended spiral inductors and intelligent microswitches/duplexers [6].

A simple technology for bulk-micromachined accelerometers [7] based on bond and etch back silicon on insulator (BESOI) wafers is described. This technology is an easy combination of bulk- and surface-micromachining technology using the buried oxide as a sacrificial layer allowing a precise control of the thickness of the beams and the fabrication of complex structures. Cantilever-beam, quad-beam, twin-mass and a new triaxial accelerometer have been fabricated and their results are described. Over-range structures have been included without any additional process step. The devices are anodically bonded to a glass wafer in order to reduce the package stresses and to control the damping of the structures.

A CMOS integrated [8] surface-micromachined angular rate sensor utilizing an electroformed vibrating metal ring structure on a silicon IC has been developed using SOI. Substantial signal-conditioning circuitry is included on the IC with the vibrating structure.

An asymmetric micro-mirror excited by an external piezoelectric ceramic vibrating element [9] directs an optical beam at an over-30-degree beam-scanning angle, Fig. 2. Using an SOI process simplifies the fabrication process and results in a very flat mirror surface. A struc-
(1)

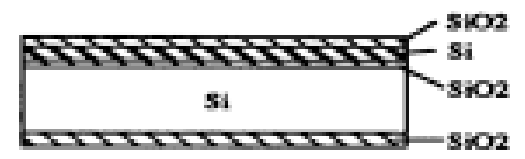

(2)

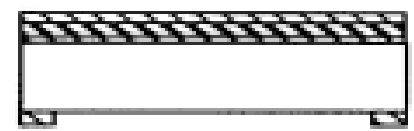

(3)

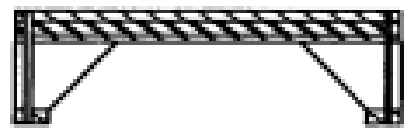

(4)

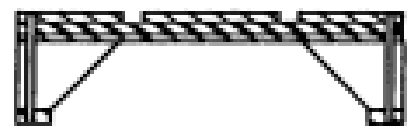

(5)

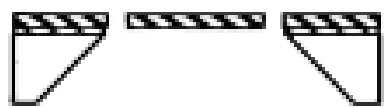

Fig. 2. Silicon micro-mirror fabrication process on SOI [9].

ture consisting of two such mirrors (one horizontal and one vertical) was used to reproduce half-VGA computer images. Full displays should be possible by adding simple signal processing.

A silicon on insulator pressure sensor with enhanced performance [10] has been developed for use at very high temperatures. A Diffusion Enhanced Fusion (DEF) bonding technique has been employed for the formation of SOI wafers. This technique simultaneously allows for the bonding of sensing gages to the dielectrically isolated silicon diaphragm and for the improvement in mechanical electrical performance of the bonded sensor. The following improvements are obtained:

1) reduced thermal shift $(1-2 \mathrm{mV}$ over the temperature range from $420^{\circ} \mathrm{C}$ to $3700^{\circ} \mathrm{C}$ ).

2) better thermal stability attributed to close thermal matching of the gages to the underling diaphragm (mechanical and electrical stability).

3) better passivation for high temperature operability. The SOI sensor structure obtained through DEF bonding approaches the optimum that can be achieved for a high temperature piezoresistive silicon pressure sensor.

A high temperature pressure sensor has been developed for measuring the cylinder pressure in combustion engines of automobiles [11]. The sensor is made of a membrane based piezoresistive $\mathrm{B}-\mathrm{SiC}$-on-SOI (SiCOI) sensor chip and a specially designed housing. The sensitivity of the sensor at room temperature is approximately $0.19 \mathrm{mV} / \mathrm{bar}$ and decreases to about $0.12 \mathrm{mV} / \mathrm{bar}$ at $300{ }^{\circ} \mathrm{C}$.

Mirrors and micromirror arrays [12] made of monocrystalline silicon are described. Electrostatically operated two-directionally deflecting mirrors and mirror arrays 


\section{A. Y. Usenko et. al.: Silicon-on-insulator technology for ...}

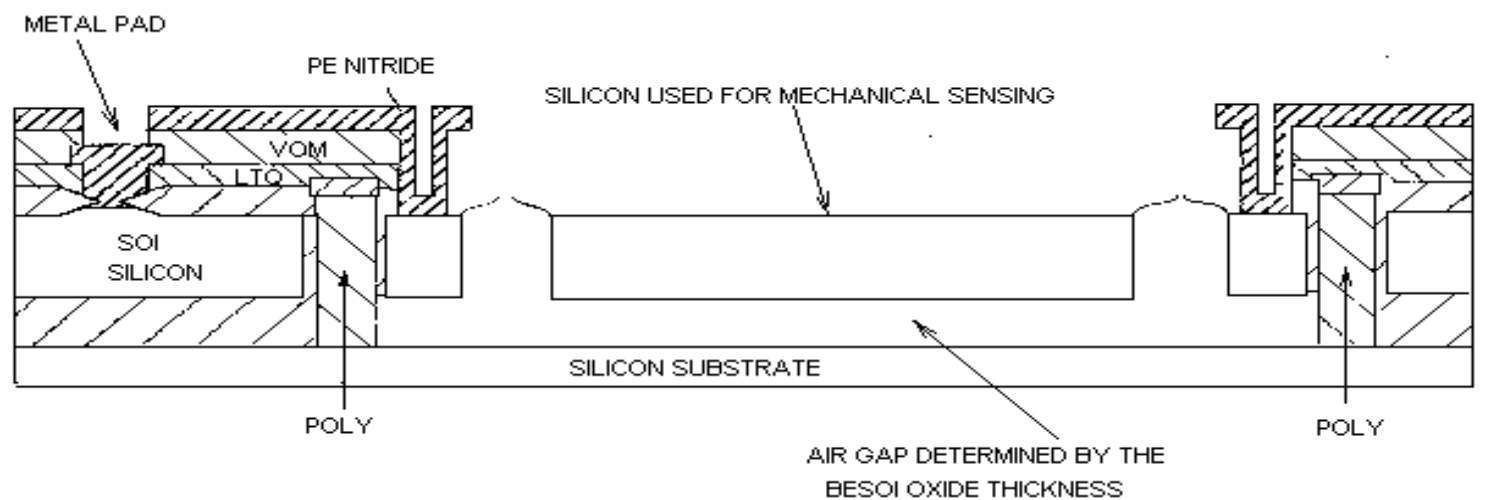

Fig. 3. Simplified cross-section of microaccelerometer on SOI [14].

for continuous scanning with working frequencies between several $100 \mathrm{~Hz}$ and $200 \mathrm{kHz}$ are presented. The modified BESOI technology used and the experimentaldata-based method to improve the accuracy of model parameters for simulations and to determine the crosscoupling between array cells are new in the field of micromechanics.

Accurate thermal simulations of SOI transistors and cantilever MEMS structures are described in [13] where lateral thermal conduction in the silicon device layer is determined by MEMS. The thermal conductivity decreases with decreasing layer thickness, $d_{s}$, to a value nearly 40 percent less than that of bulk silicon for $d_{s}=0.42 \mu \mathrm{m}$. The data show that the buried oxide in BESOI wafers has a thermal conductivity that is nearly equal to that of bulk fused quartz.

A surface micromachined accelerometer using SOI
[14] is described, Fig. 3. Both the acceleration (or deceleration) sensor and associated signal conditioning circuitry are monolithically fabricated on the same substrate. The top silicon layer of the SOI wafer is the movable, common electrode of a differential capacitor pair. The components of the signal conditioning circuitry are fabricated in the SOI layer. The top silicon layer is single crystal silicon and does not suffer from the stress-related warping so common with polysilicon.

Micromachined, thermally insensitive silicon resonators with accuracy equivalent or superior to that of quartz resonators are fabricated from a micromechanical SOI process in [15]. A resonator forms a tuning fork gyroscope. Radiation-hard precision voltage references are made using the silicon resonators. Thermal stability is comparable to that of a quartz oscillator. By employing a micromechanical device based in a tuning fork gyro-

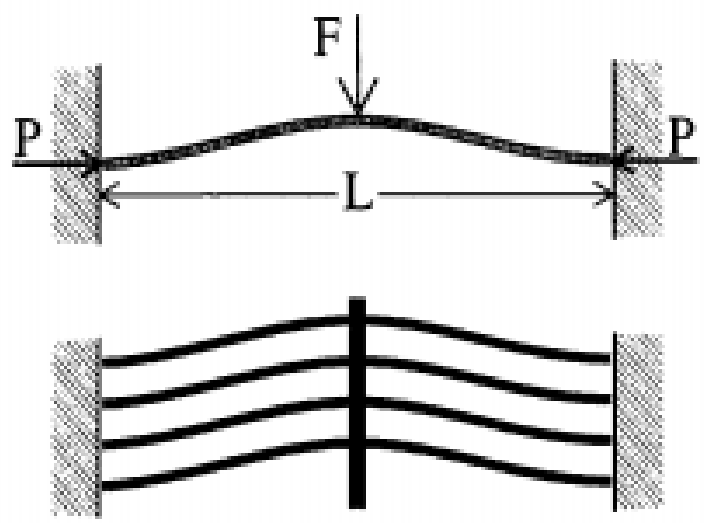

(a) A single buckled beam, (b) configuration with four beams coupled in paralel.

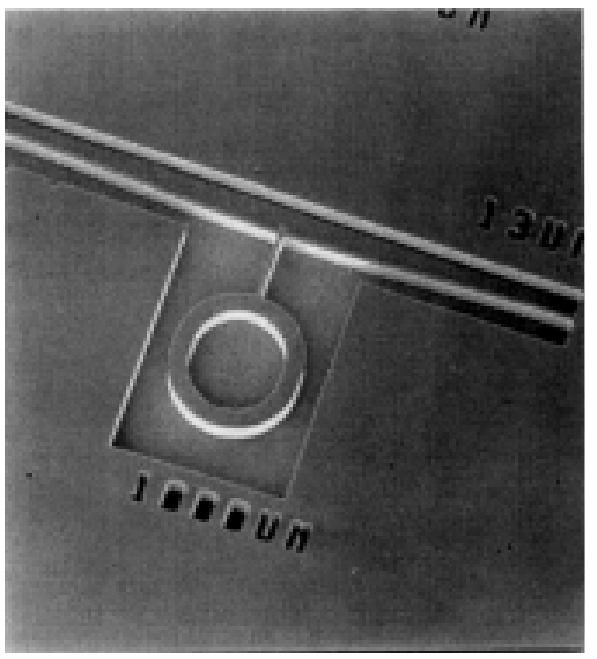

Fig. 4. Bistable buckled beam, its cross-section (left) and photograph (right) [16]. 


\section{A. Y. Usenko et. al.: Silicon-on-insulator technology for ...}

scope, resonators are made for either or both of the gyro drive and sense axes.

A lateral symmetrical bistable buckled beam for snapin holding structures is a SOI structure described in [16], Fig. 4. The structures were fabricated by deep silicon reactive ion etching using the black silicon method than subsequently released and thermally oxidized. The bistability was monitored in situ in a scanning electron microscope using a micromanipulator.

$\mathrm{KOH}$ solutions for etching extremely sharp silicon tips on SOI is described in [17]. Etching of (100) silicon on SOI wafers was carried out over a wide range of reaction temperatures and $\mathrm{KOH}$ concentrations. The sharpness increases with temperature to a critical point and then decreases at very high temperature and $\mathrm{KOH}$ concentration. Hydrogen bubbles formed during etching are very important in determining both etch rate and sharpness of the tips. The sharpest tip dimension was found to occur at $30 \% \mathrm{KOH}$ and $70{ }^{\circ} \mathrm{C}$ reaction temperature.

A process for further reducing dimensions to a nanometer-scale for SOI MEMS has been demonstrated in [18]. Substrates was patterned using high resolution electron beam lithography with $100 \mathrm{keV}$ electrons followed by $\mathrm{Al}$ evaporation and liftoff. The $\mathrm{Al}$ is used as an etch mask in $\mathrm{CF}_{4}$ reactive ion etching to pattern the top silicon layer. Then structures were undercut using a buffered oxide etch. The structures were made from substrates having a top silicon thickness of 200 or $50 \mathrm{~nm}$, and a buried oxide thickness of $400 \mathrm{~nm}$. With this process a variety of movable structures have been made. The performance of an electrostatically driven FabryPerot interferometer that consists of a $\mathrm{M}$ sized platform suspended by wires that are $100-200 \mathrm{~nm}$ wide is described. Much smaller mechanical structures such as suspended silicon beams as narrow as $30 \mathrm{~nm}$ have also been made.

A new form of rotating media disk drive is described [19] using extremely sharp silicon tips. Data storage densities of many gigabits per square inch can be obtained. Data is stored in localized regions of electric charge that are stored within a thin dielectric layer that is coated upon both sides of an electrically conductive platter, rather than in magnetic domains. Data is read and written via ungated field emitter tips rather than with transformers and magnetically sensitive elements. The read/write head is built from the read/write head chip that contains field emitter tips and other elements. This chip is an active CMOS or other circuit whose active area is parallel to one side of a platter, rather than having a single, passive write element and a single, passive read element with external control circuits and amplifiers. The read/write head is a CMOS SOI with the tips and other microelectromechanical elements being formed in the top, silicon membrane, on isolated islands or pillars, and the supporting circuitry being formed in the base silicon wafer. Each read/write head chip is attached to the end of the arm of an actuator, in a manner similar to that used in magnetic disk drives.

\section{Acknowledgement}

This work is partially funded by US Ballistic Missile Defense Organization Contract № DASG60-98-M-0127.

\section{References}

1. S. Renard, A. Auberton-Herve, Silicon-On-Insulator Micromachining //European Semicond. Design Product.Assembly 20, 13 (1998).

2. Q.-Y. Tong, U. Goesele// in Semiconductor wafer bonding: Science and Technology p. 252, John Wiley \& Sons, NY (1998).

3. M. Schmidt, Wafer-to-wafer bonding for microstructure formation // Proceedings of the IEEE, 86, 1575 (1998).

4. A. Madni, L. Wan, Microelectromechanical systems (MEMS): An overview of current state-of-the-art //in Proceedings of the IEEE Aerospace Conference. Part 1 (of 5) Mar 21-28 1998, 11998 p. 421, Snowmass at Aspen, CO, USA (1998).

5. H. Nakanishi, T. Nishimoto, R. Nakamura, A. Yotsumoto, Studies on $\mathrm{SiO} 2-\mathrm{SiO} 2$ bonding with hydrofluoric acid - room temperature and low stress bonding technique for MEMS// in Proc. of the IEEE Annual International Workshop on Micro Electro Mechanical Systems p. 609, Jan 25-29 Heidelberg, Germany (1998).

6. P. K. Vasudev, Telecom Explosion Drives RF Ics // Electronic Engineering Times, July 13, p.81 (1998).

7. J. +A. Plaza, J. Esteve, Simple technology for bulk accelerometer based on bond and etch back silicon on insulator wafers // Sensors and Actuators A:Phys. 68, 299 (1998).

8. S. Chang, M. Chia, P.et all, CMOS integrated angular rate sensor // Sensors and Actuators, A: Physical, 66 p. 138 (1998).

9. K. Yamada, T. Kuriyama, Novel asymmetric silicon micro-mirror for optical beam scanning display // Proceedings of the IEEE Annual International Workshop on Micro Electro Mechanical Systems p. 110, Jan 25-29 1998 Heidelberg, Germany (1998).

10. A. Ned, A. Kurtz, High temperature, silicon on insulated silicon pressure sensors with improved performance through Diffusion Enhanced Fusion (DEF) bonding // Proc. of the Int. Instrumentation Symp. May 3-7 1998 Reno, NV, p. 120, (1998).

11. Jvon Berg, R. Ziermann, W. Reichert, E. Obermeier, M. Eickhoff, G. Kroetz, U. Thoma, Th. Boltshauser, C. Cavalloni, J. P. Nendza, (1998) High temperature piezoresistive в-SiC-on-SOI pressure sensor for combustion engines // Materials Science Forum. Part 2 (of 2) 264-268, 1101 (1998).

12. S. Kurth, R. Hahn, C. Kaufmann, K. Kehr, J. Mehner, U. Wollmann, W. Doetzel, T. Gessner, Silicon mirrors and micromirror arrays for spatial laser beam modulation // Sensors and Actuators, A: Physical 66, 76 (1998).

13. M. Asheghi, M.N. Touzelbaev, K.E. Goodson, Y.K. Leung, S.S.: Wong Temperature-Dependent Thermal Conductivity of Single-Crystal Silicon Layers in SOI Substrates // Journal of Heat Transfer, Transactions ASME, 120, 30 (1998).

14. R. Bashir and A. Kabir Method of making surface micro-machined accelerometer using silicon-on-insulator technology // United States Patent 5,747,353 May 5 (1998).

15. M. Weinberg P. Ward and A. Kourepenis, Temperature insensitive silicon oscillator and precision voltage reference formed therefrom US Patent 5,783,973 Jul. 21 (1998).

16. M. Vangbo and Y. Вдcklund, A lateral symmetrically bistable buckled beam // J. Micromech. Microeng. 8, 129(1998).

17. M. H Yun, V. A. Burrows, and M. N. Kozicki, Analysis of KOH etching of (100) silicon on insulator for the fabrication of nanoscale tips // J. of Vacuum Sci. \& Technol. B 16, 2844 (1998).

18. D. W. Carr and H. G. Craighead, Fabrication of nanoelectromechanical systems in single crystal silicon using silicon on insulator substrates and electron beam lithography // J. of Vacuum Sci. \& Technol. B 15, 2760 (1997).

19. S. G. Morton, Tera-byte disk drive US Patent 5,724,336 Mar. 3 (1998). 